\title{
High-throughput chromosome conformation capture-based analysis of higher-order chromatin structure in nasopharyngeal carcinoma
}

\author{
Yuanyuan Yang ${ }^{1 \#}$, Mingfa Chen ${ }^{2 \#}$, Lingjun Cheng ${ }^{1}$, Canping $\mathrm{Su}^{1}, \mathrm{Xiyi}_{\mathrm{Liao}}{ }^{3}, \mathrm{Hong} \mathrm{zhang} \mathrm{He}^{1}$, \\ Mingming You ${ }^{1}$, Gang Rui ${ }^{4} \wedge$, Guolin Hong ${ }^{1 \wedge}$
}

${ }^{1}$ Department of Laboratory Medicine, The First Affiliated Hospital of Xiamen University, Xiamen Key Laboratory of Genetic Testing, Xiamen, China; ${ }^{2}$ Nanping Maternal and Child Health Hospital of Fujian Province, Nanping, China; ${ }^{3}$ Department of Radiation Oncology, Xiamen Cancer Hospital, The First Affiliated Hospital of Xiamen University, Xiamen, China; ${ }^{4}$ Department of Orthopedic Surgery, The First Affiliated Hospital of Xiamen University, Xiamen, China

Contributions: (I) Conception and design: Y Yang, M Chen, G Hong; (II) Administrative support: Y Yang, M Chen, G Rui, G Hong; (III) Provision of study materials or patients: Y Yang, M Chen, L Cheng, C Su, G Hong; (IV) Collection and assembly of data: Y Yang, M Chen, L Cheng, C Su, G Hong; (V) Data analysis and interpretation: Y Yang, M Chen, X Liao, H He, M You, G Hong; (VI) Manuscript writing: All authors; (VII) Final approval of manuscript: All authors.

\#These authors contributed equally to this work.

Correspondence to: Gang Rui. Department of Orthopedic Surgery, The First Affiliated Hospital of Xiamen University, 55 Zhenhai Road, Siming, Xiamen 361005, China. Email: reigang@163.com; Guolin Hong. Department of Laboratory Medicine, The First Affiliated Hospital of Xiamen University, Xiamen Key Laboratory of Genetic Testing, 55 Zhenhai Road, Siming, Xiamen 361005, China. Email: xmhg19899@sina.com.

Background: Firstly, we aimed to compare the differences of higher-order chromatin structure between nasopharyngeal carcinoma (NPC) and normal nasopharyngeal tissues. The second objective was to analyze the specific chromatin interaction site of NPC and the NPC-related genes regulated by this interaction site.

Methods: We included 6 NPC patients and 6 healthy controls to obtain the sequencing results of highestthroughput chromosome conformation capture (Hi-C) technique, followed by further analysis of the specific chromatin interaction sites in NPC.

Results: We found an abnormal ultra-long distance interaction site on the chromosome 7p in the CNE210 sample, which was caused by a fusion gene SEPT7P2-PSPH. Additionally, a significant interaction site between chromosome 8q and 3p was revealed in the samples CNE25, CNE29, and CNE211, which was the interaction between $1.5 \mathrm{~kb}$ downstream of $A S A P 1$ and $0.8 \mathrm{~kb}$ upstream of $C T N N B 1$ gene. Further quantitative polymerase chain reaction (qPCR) revealed that $A S A P 1$ and $C T N N B 1$ genes were more highly expressed in CNE25, CNE29, and CNE211 than in the Np group, preliminarily indicating that this interaction site was likely related to the high expression of ASAP1 and CTNNB1 in NPC.

Conclusions: Through Hi-C analysis, we analyzed the specific chromatin interaction sites associated with NPC, and found the chromosomal translocation and chromatin interaction sites associated with NPC based on statistical analysis. This study has certain guiding significance for in-depth study of the mechanism of NPC occurrence and development.

Keywords: Hi-C analysis; Hi-C library preparation; nasopharyngeal carcinoma (NPC); chromatin interaction

Submitted Jun 07, 2021. Accepted for publication Jul 27, 2021.

doi: $10.21037 /$ atm-21-3273

View this article at: https://dx.doi.org/10.21037/atm-21-3273

^ ORCID: Gang Rui, 0000-0003-4919-1648; Guolin Hong, 0000-0002-4812-098X. 


\section{Introduction}

Nasopharyngeal carcinoma (NPC), as a common malignant tumor of the head and neck (1) and it has significant geographical and ethnic differences in its incidence. In 2020, there were 133,354 newly diagnosed NPC patients worldwide (2). At present, the etiology and pathogenesis of NPC remain unclear, and the treatment of NPC is still based on radiotherapy and chemotherapy. Generally, NPC patients are found to progress to late stage, leading to poor prognosis and treatment outcomes (3-5). Therefore, there is an urgent need to investigate and elucidate the pathogenesis of NPC.

According to previous clinical studies, mutations in some genetic information are closely related with NPC development (6). Chromosomes are carriers of genetic information, which occupy independent nuclear regions in a highly organized way rather than random structures (7). Chromosome conformation reflects and also probably regulates basic biological processes (8). Highestthroughput chromosome conformation capture (HiC) takes the whole nucleus as the research object. This technology utilizes high-throughput sequencing combined with bioinformatic methods to study genome-wide chromosomal interactions, in order to obtain the genome sequence and chromatin three-dimensional structure information at the chromosome level, which are crucial for the normal function of cells (9-11).

A genomic contact matrix produced by $\mathrm{Hi}-\mathrm{C}$ analysis, at a specific resolution given by sequencing depth, measures the strength of interaction between 2 genomic loci (12). The combination of Hi-C analysis and chromatin coimmunoprecipitation sequencing (ChIP-Seq) with transcriptome analysis can elaborate the mechanisms involved in trait formation in organisms from gene regulatory networks and epigenetic networks. Human genome chromatin is highly folded within a narrow nucleus and there are some interactions and regulatory relationships within and between chromatin (13). However, studies on specific chromatin interaction sites in NPC are still limited. In this study, by utilizing $\mathrm{Hi}-\mathrm{C}$ technology, an important method to study the spatial structure of chromatin, we aimed to analyze and compare the differences in the higherorder chromatin structure between NPC and adjacent tissues, and explore the specific chromatin interaction sites of NPC to further investigate the NPC related genes regulated by the interaction sites.

We present the following article in accordance with the MDAR reporting checklist (available at https://dx.doi. org/10.21037/atm-21-3273).

\section{Methods}

\section{General information}

Biopsy tissue samples were obtained from 6 healthy controls (Np group) and 6 NPC patients (CNE group) admitted to the First Affiliated Hospital of Xiamen University between February 2017 and December 2018. All participants provided written informed consent, and the study was conducted with the approval of the Ethics Committee of The First Affiliated Hospital of Xiamen. All procedures performed in this study involving human participants were in accordance with the Declaration of Helsinki (as revised in 2013).

\section{Study methods}

\section{Sample sequencing}

Fresh specimens were fixed and cross-linked with $37 \%$ formaldehyde according to known methods, followed by cell lysis. Then, the quality of the samples was examined, and those deemed to be good quality were utilized for Hi-C fragment preparation. Chromatin digestion was performed using restriction endonuclease (HindIII/MboI) and the digestion effect was examined. Next, on completion of biotin labeling, blunt-end ligation, and DNA purification, Hi-C samples were prepared and subsequently taken for DNA quality testing. The samples that passed the DNA quality test were utilized for standard library preparation. On completion of removal of biotin, sonication, end repair, and "A" tailing, the fragments containing biotin were captured and bound to ligated adapters, and eventually Hi-C libraries were obtained after polymerase chain reaction (PCR) amplification and a final quality control. The next sequencing step was completed utilizing a HiSeq 3000 sequencing system (Illumina, San Diego, CA, USA). The specimens were further analyzed for the overall understanding of intrachromosomal (cis-acting) and interchromosomal (trans-acting) and NPC specific chromatin interaction sites. Sample information is shown in Table 1.

\section{Quality analysis of sequencing data}

The sequencing error rate was related to base quality and was also affected by multiple factors such as the sequencer 
Table 1 Sample information

\begin{tabular}{|c|c|c|c|}
\hline Sample name & Latin name of species & Sample type & Endonuclease \\
\hline Np6915 & Homo sapiens & $\mathrm{Np}$ & Mbol \\
\hline CNE29 & Homo sapiens & CNE & Mbol \\
\hline CNE210 & Homo sapiens & CNE & Mbol \\
\hline Np691 & Homo sapiens & $\mathrm{Np}$ & Mbol \\
\hline Np692 & Homo sapiens & $\mathrm{Np}$ & Mbol \\
\hline Np693 & Homo sapiens & $\mathrm{Np}$ & Mbol \\
\hline CNE24 & Homo sapiens & CNE & Mbol \\
\hline Np697 & Homo sapiens & $\mathrm{Np}$ & Mbol \\
\hline
\end{tabular}

Table 2 Corresponding relationship of base identification and Phred score

\begin{tabular}{lccc}
\hline Phred score & Error & Accuracy & Q-score \\
\hline 10 & $1 / 10$ & $90 \%$ & Q10 \\
20 & $1 / 100$ & $99 \%$ & Q20 \\
30 & $1 / 1,000$ & $99.90 \%$ & Q30 \\
40 & $1 / 10,000$ & $99.99 \%$ & Q40 \\
\hline
\end{tabular}

itself, sequencing reagents, and samples. The base quality could be measured by the Phred score (or Q score) which was calculated with the following formula:

$$
\text { Phred }=-10 \log _{10} e
$$

The Phred score estimated the probability of incorrect base call with "e" as an error rate. The corresponding relationship is shown in Table 2.

\section{Base distribution analysis of sequencing data}

The base distribution of reads was obtained with the base position of the filtered reads sequence as the abscissa, and the proportion of adenosine thymine cytosine guanine (ATCG) bases and N (unidentified bases) at each position as the ordinate.

\section{Analysis of cis-trans interaction proportion}

Cis refers to paired reads on the same chromosome, and trans refers to paired reads on different chromosomes. In the genome-wide contact matrix, this pattern is characterized by significant intra-chromosomal interactions and fewer interchromosomal interactions along the diagonal. Part of the reason for this pattern is that chromosomes are physically separated in the nucleus and occupy independent nuclear regions. This pattern can simply evaluate the quality of data. The existence of noise in the matrix, such as random noise, may cause cis and trans interactions to be similar. A high-noise experiment will also lead to similar proportions of cis and trans interactions. Therefore, this pattern is usually quantified through a statistical ratio of cis-trans to total interaction (14). On completion of the calculation of genomic distance $\mathrm{S}$ (distance between nucleotide sequences in base pairs) and chromosome interaction frequencies (IFs), their logarithms were taken for mapping.

\section{Establishment of contact matrix}

The Hi-C contact matrix refers to a list of DNA-DNA contacts generated using $\mathrm{Hi}-\mathrm{C}$ data, which represents the actual interaction probability of various sites in the genome (15). Genome-wide contact matrix can be constructed by efficient statistics of all contacts of the whole genome, which can be visualized by the software HiC-Pro as a heat map, that is, the Hi-C map (16). The resolution of Hi-C map is defined as: when a contact matrix is constructed with the minimum unit length, $80 \%$ of the unit length contains a minimum of over 1,000 contacts, and this minimum unit length is the resolution (17). Higher 


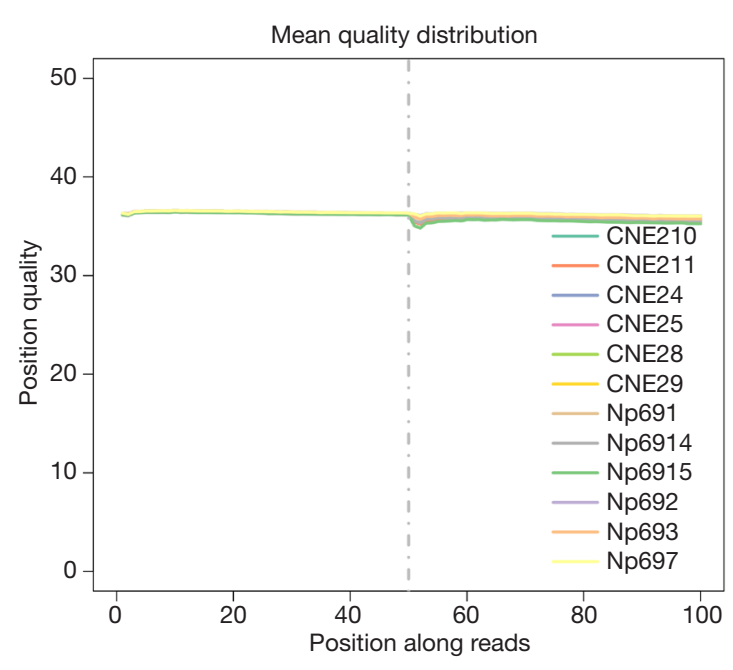

Figure 1 Sequencing quality distribution map.

resolution reflects smaller scope of the genome.

Lieberman-Aiden's study on Hi-C found that the mammalian genome can be divided into 2 chromosomal structural regions called A/B compartments; much of the interaction between loci occurs in the same chromosomal structural region (18). Further, studies have revealed closer relations of A compartment with open chromatin, and of B compartment with closed chromatin. The A/B compartments are cell-specific and can converse in different tissues and cells, this conversation partly relates to gene expression regulation (19). Therefore, analysis of $\mathrm{A} / \mathrm{B}$ compartments can determine intra- and inter-chromosomal interactions.

\section{Calculation of topologically associated domains (TAD)- fusion score}

In a mammalian Hi-C map with about $40 \mathrm{~kb}$ resolution, a distinct triangular shape is presented, and this structure is named TAD (20) with significant boundaries among domains. It has been shown that CTCF and structural proteins such as cohesin significantly affect the formation of TAD boundaries (21). The CTCF is a repressive transcription factor that directionally binds specific DNA sequence sites (22), and it mediates insulator looping with off-target genes while disrupting enhancer looping, thereby inhibiting transcription of genes $(23,24)$.

Servant introduced the TAD-fusion score to quantitatively describe that genomic fragment deletion is more likely to cause TAD-fusion, subsequently cause abnormal enhancer-promoter interactions, and ultimately diseases (16). This score is a measure of the degree of compactness of TAD. The more compact the TAD, the stronger the interaction inside the TAD and the weaker the interaction among the TAD (25). The TAD-fusion score = internal contacts/external contacts.

\section{Software}

The software Bowtie2 (Johns Hopkins University, Baltimore, MD, USA) and HiC-Pro (26) were used to generate the reference sequences and process the $\mathrm{HiC}$ data.

\section{Results}

\section{Sequencing quality distribution map}

The sequencing quality distribution map was obtained with the base position of the filtered sequence as the abscissa and the average quality value of each position as the ordinate, in order to roughly reflect the stability of sequencing quality during sequencing (Figure 1). The map showed that the overall sequencing quality of the 12 specimens was qualified, and base call accuracy was above $99.9 \%$.

\section{Base distribution map}

As shown in Figure 2, there was an equal proportion of A and $\mathrm{T}$ bases and an equal proportion of $\mathrm{G}$ and $\mathrm{C}$ bases at each position of the Reads during sequencing. This result indicated no sequence bias, and the base distribution of the samples was in line with the expectation.

\section{Cis-trans interaction proportion}

Analysis of the proportion of cis-trans interaction showed that the cis-acting sites were greater than $80 \%$ in all 12 specimens (Figure 3), indicating that the cis-acting was greater than the trans-acting in both $\mathrm{CNE}$ and $\mathrm{Np}$ groups.

\section{Cis-trans IFs}

The IFs obtained from Hi-C-based experiments generally decay with increasing distance in a given range, and the slopes of IFs could be described by the interaction decay exponents (IDEs) (18). The result showed (Figure 4) that cis-trans IFs decreased significantly with increasing sequence space. 


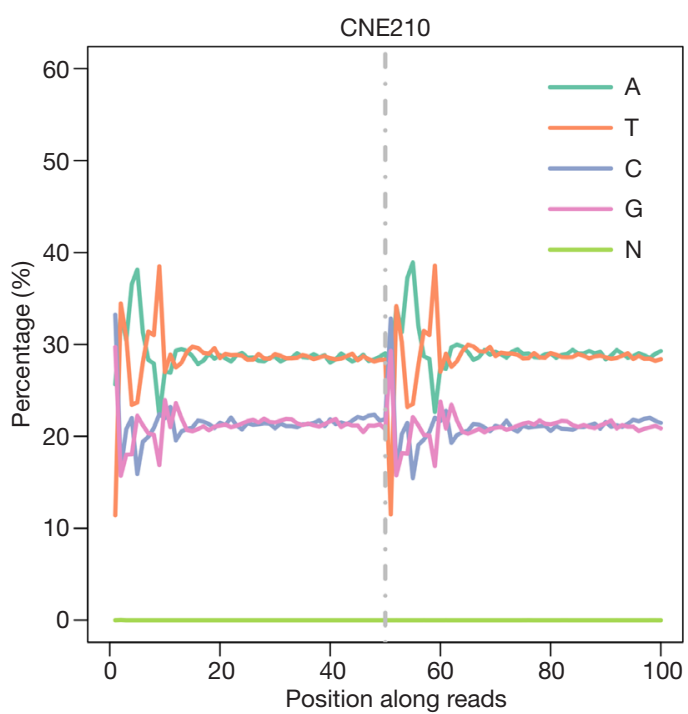

Figure 2 Base distribution map.

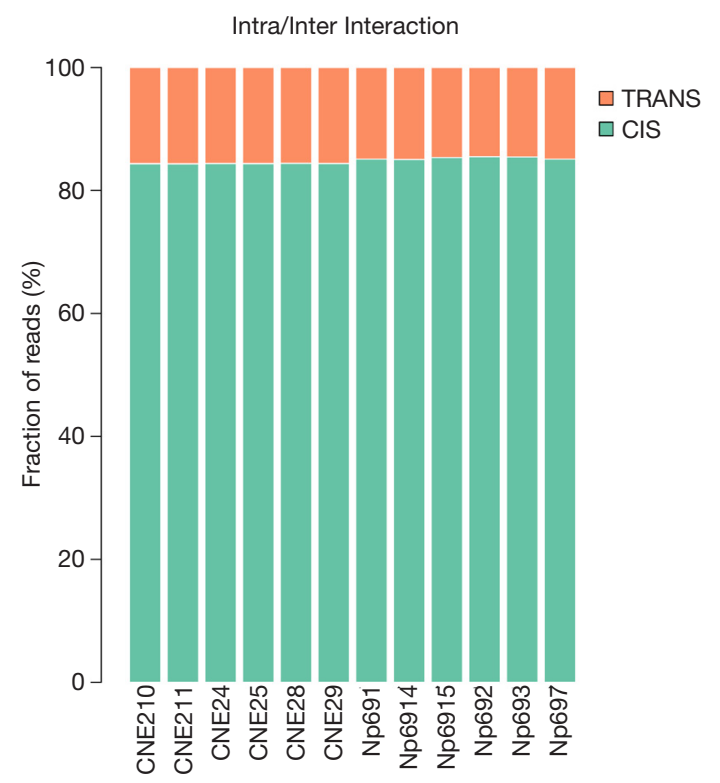

Figure 3 Cis-trans interaction proportion.

\section{Chromosome interactions}

\section{Intrachromosomal interactions}

From yellow to red, intrachromosomal interaction intensity was gradually increased (Figure 5). The result showed an abnormal ultra-long distance interaction site on the chromosome $7 \mathrm{p}$ in the CNE210 sample, which was caused by a fusion gene, SEPT7P2-PSPH, that has been previously reported (27).

\section{Interchromosomal interactions}

Although chromosomes occupy independent nuclear regions, there are interchromosomal interactions due to the small space of the nucleus. It has been found that in human and murine cells, long chromosomes tend to be closer to long chromosomes, and short ones to short ones (28). After normalizing the interaction between chromosomes, we obtained the contact matrix represented as a heatmap. The higher value of the interchromosomal interaction, the closer the 2 chromosomes in space. As shown in Figure 6, a significant interaction site between chromosome $8 \mathrm{q}$ and $3 \mathrm{p}$ was found in the samples CNE25, CNE29, and CNE211, which was the interaction between $1.5 \mathrm{~kb}$ downstream of $A S A P 1$ and $0.8 \mathrm{~kb}$ upstream of CTNNB1 gene. The quantitative polymerase chain reaction (qPCR) revealed that $A S A P 1$ and $C T N N B 1$ genes were expressed higher in CNE25, CNE29, and CNE211 than in the Np group.

\section{Genome-wide interactions}

A genome-wide contact matrix was constructed on the sequences obtained from Hi-C sequencing, and then a Hi-C map was obtained after normalization (Figure 7). The color of each point represents the log value of interaction intensity of the corresponding genomic loci. From yellow to red, interaction intensity was gradually enhanced. From the obvious red diagonal in the Figure 7, we could see that the interaction of homologous chromosomes was stronger than that of non-homologous chromosomes, and the intrachromosomal interaction was also stronger than the interchromosomal interactions.

\section{Distribution of TAD-fusion score}

We randomly selected a region of the chromosomes of 12 samples to construct an interactive heatmap and subsequently to calculate the TAD-fusion score. The comparison of the scores of 12 samples was used to determine whether the compactness of the TAD changes under different conditions. As shown in Figure 8 , in comparison with the $\mathrm{Np}$ group, higher fusion score was identified in the CNE group, indicating a significant TAD sliding in NPC.

\section{Discussion}

Currently, NPC treatment is still based on traditional chemotherapy, and there is a lack of specific targeted agents for NPC. In recent years, the development of next- 


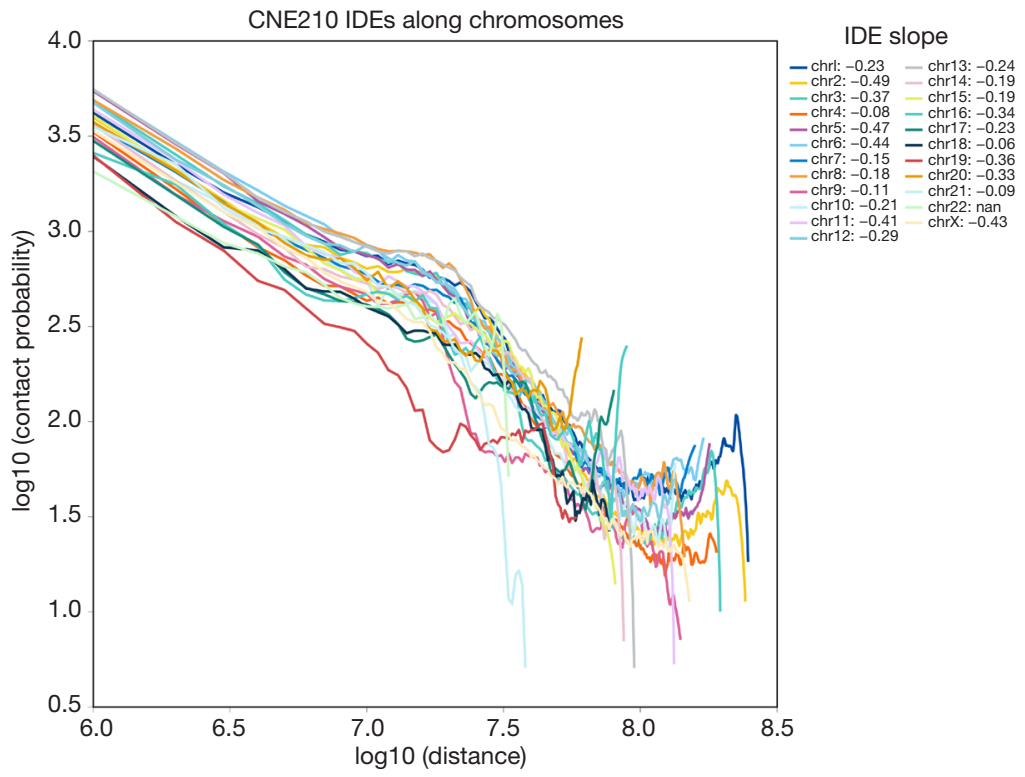

Figure 4 Cis-trans interaction frequencies. Abscissa: interchromatin distance at different sites; ordinate: interaction strength.

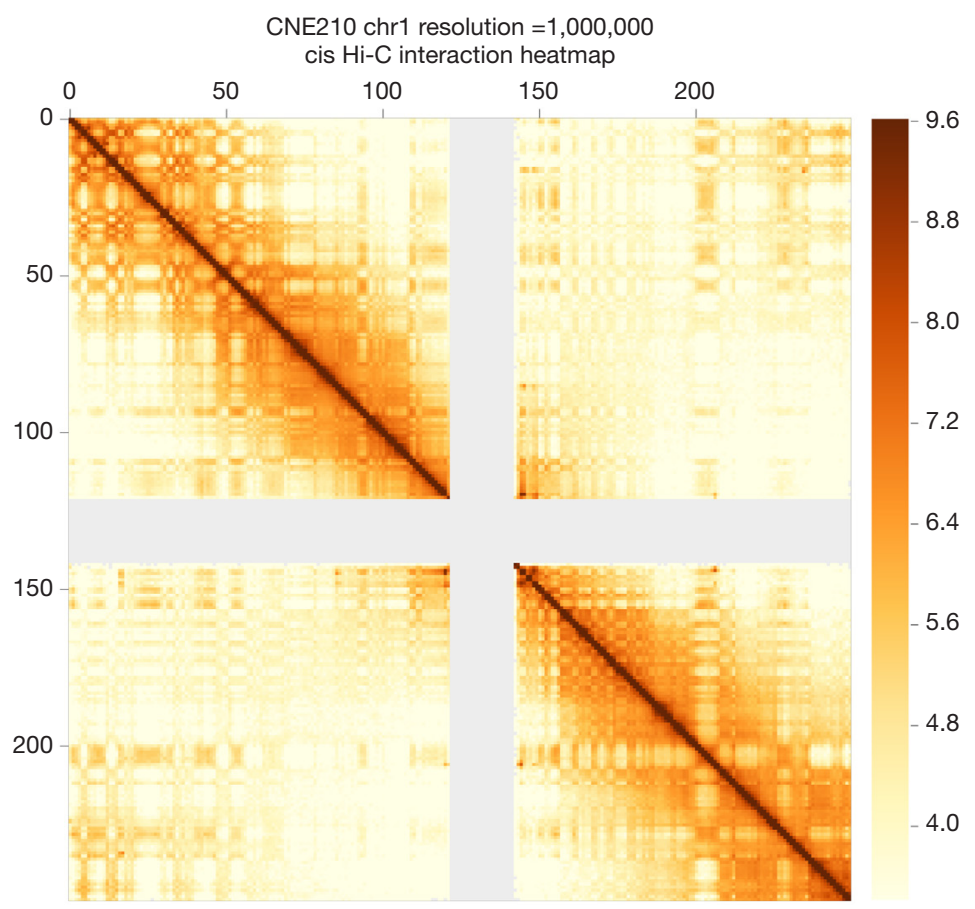

Figure 5 Heatmap of intrachromosomal interactions.

generation sequencing technology has greatly promoted the study of genes, transcriptome, and epigenome in human cancers. In this study, on completion of Hi-C library preparations, sequencing, and establishment of a contact matrix, a genome-wide interaction heatmap was obtained through use of the latest $\mathrm{Hi}-\mathrm{C}$ technology. Our results were further analyzed based on existing studies, so as to explore specific chromosome interaction sites in NPC and provide 


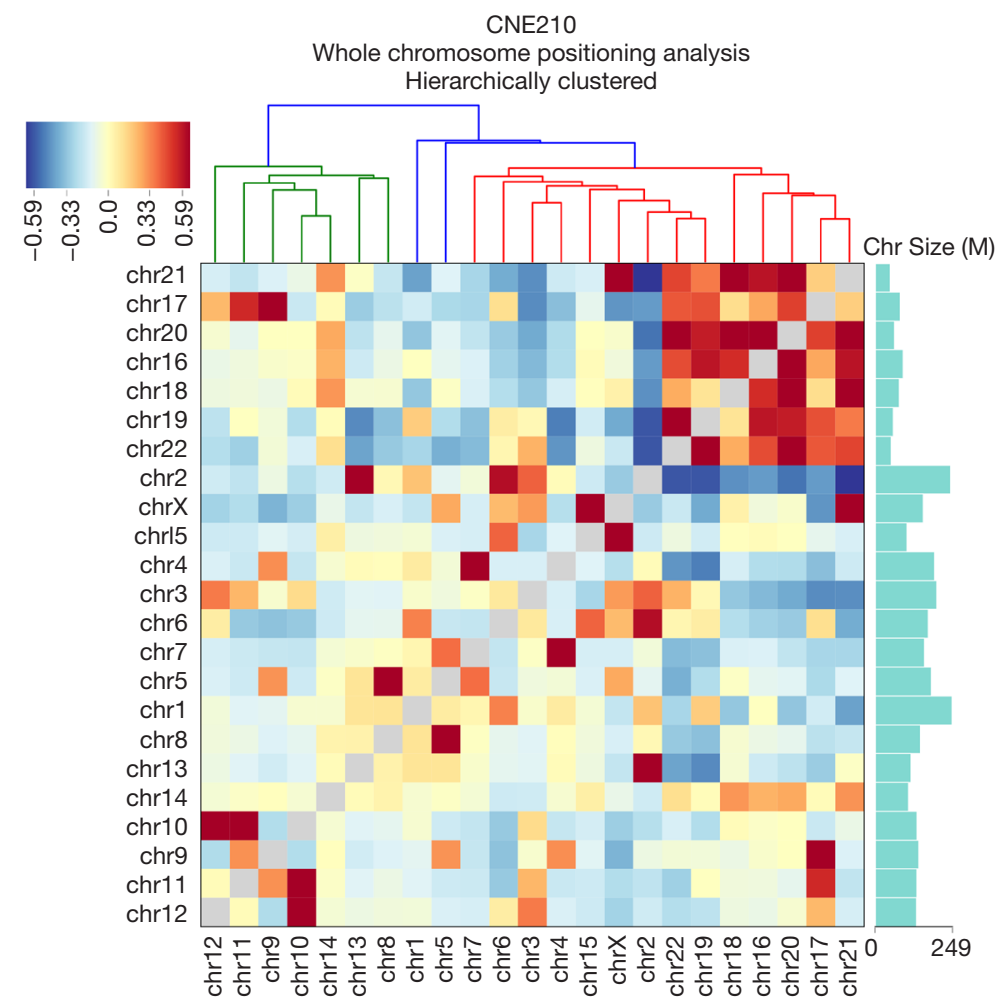

Figure 6 Heatmap of interchromosomal interactions after normalization.

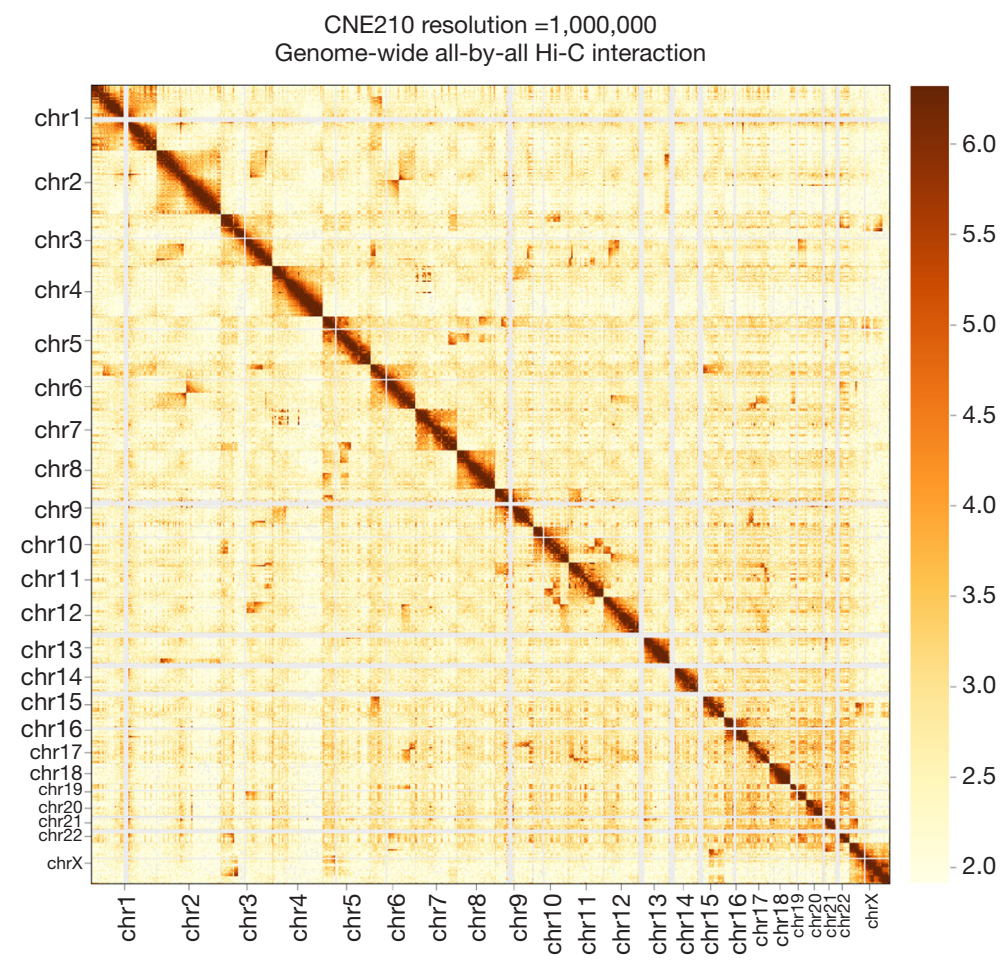

Figure 7 Heatmap of genome-wide interactions. 


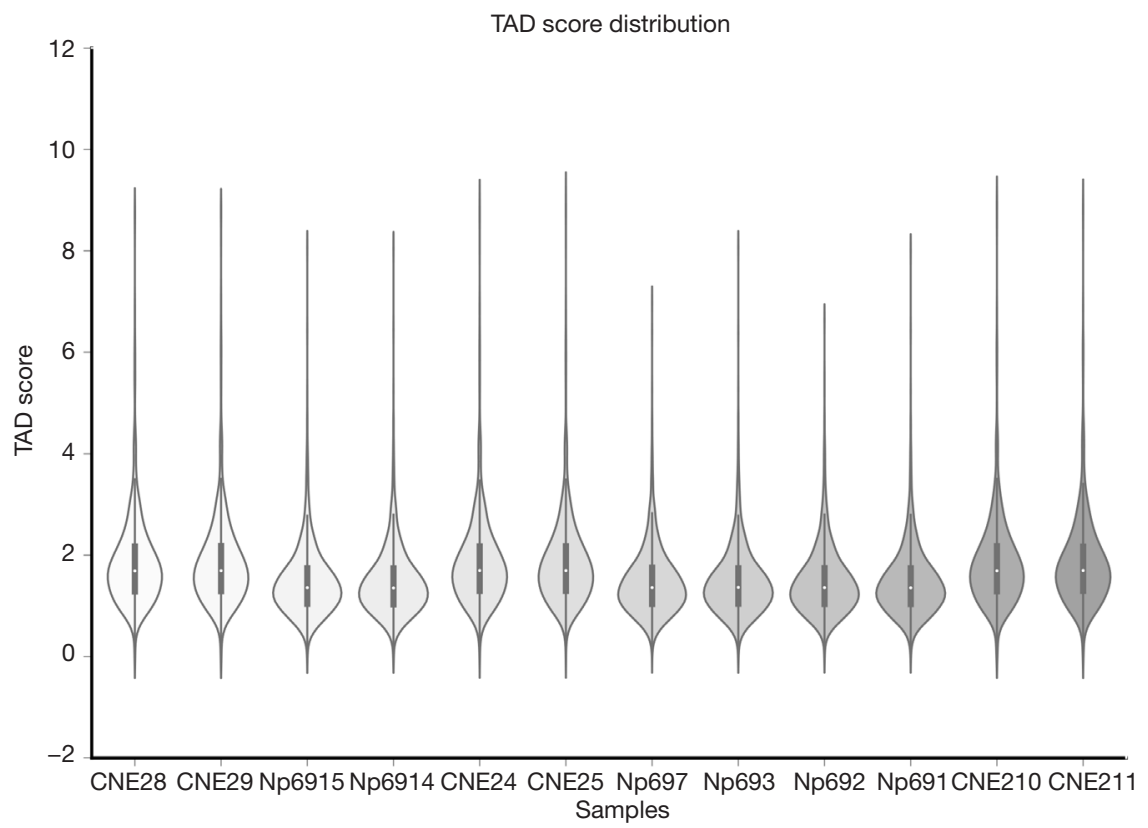

Figure 8 TAD score distribution of each sample. TAD, topologically associated domains.

a reliable experimental basis for clinical studies on NPC pathogenesis and targeted therapy.

A study by Zhang et al. (29), with paraffin-embedded samples from 127 NPC patients, found that HOXC6 expression was significantly correlated with $\mathrm{T}$ stage, $\mathrm{N}$ stage, and clinical stage of NPC. Lu et al. (30) reported that EVIlexpression was increased in NPC tissues and cell lines, subsequently promoting the epithelial-mesenchymal transition of NPC cells and consequently promoting the differentiation of cancer cells. Wang et al. (31), through both in vitro and in vivo experiments, demonstrated the upregulation of TMPRSS3 expression in human NPC tissues and cell lines, and inhibition of proliferation and tumorigenicity of NPC cells by knockdown of TMPRSS3 expression. Sun et al. (32) showed the promotion of NPC cell proliferation by HOXA10. Zhang et al. (33) linked the promotion of NPC invasion and metastasis with activation of the NFAT1/ITGA6 signaling pathway by NFAT1. Li et al. (34) pointed out that measuring SLIT2 promoter methylation in blood samples was a potential diagnostic method for NPC, and the diagnostic accuracy of this method could be improved by combining multiple samples.

Our results showed that the cis-acting was greater than the trans-acting in both $\mathrm{CNE}$ and $\mathrm{Np}$ groups; cis-trans IFs decreased significantly with increasing sequence space; and there were some specific chromosome interaction sites in the NPC samples. A significant interaction site between chromosome $8 \mathrm{q}$ and $3 \mathrm{p}$ was found in the samples CNE25, CNE29, and CNE211, which was the interaction between $1.5 \mathrm{~kb}$ downstream of $A S A P 1$ and $0.8 \mathrm{~kb}$ upstream of $C T N N B 1$ gene. Further qPCR revealed that $A S A P 1$ and CTNNB1 genes were more highly expressed in CNE25, CNE29, and CNE211 than in the Np group, preliminarily indicating that this interaction site was probably related to the high expression of ASAP1 and CTNNB1 in NPC. Through Hi-C analysis, we analyzed the specific chromatin interaction sites associated with NPC, and located the chromosomal translocation and chromatin interaction sites associated with NPC based on statistical analysis. This study has certain guiding significance for in-depth study of NPC pathogenesis, but these outcomes require confirmation by further studies.

In summary, Hi-C technology can realize the screening and verification of high-throughput sequencing in large sample sizes in a short time. Therefore, it is more conducive to studies on early diagnosis and pathogenesis of cancers, and contributes to targeted drug therapy and individualized treatment for cancer patients.

\section{Acknowledgments}

Funding: We sincerely acknowledge the financial support 
of the National Natural Science Foundation of China (81772287, 81371902), and Natural Science Foundation of Fujian Province (2020J011241).

\section{Footnote}

Reporting Checklist: The authors have completed the MDAR reporting checklist. Available at https://dx.doi. org/10.21037/atm-21-3273

Data Sharing Statement: Available at https://dx.doi. org/10.21037/atm-21-3273

Conflicts of Interest: All authors have completed the ICMJE uniform disclosure form (available at https://dx.doi. org/10.21037/atm-21-3273). The authors have no conflicts of interest to declare.

Ethical Statement: The authors are accountable for all aspects of the work in ensuring that questions related to the accuracy or integrity of any part of the work are appropriately investigated and resolved. All procedures performed in this study involving human participants were in accordance with the Declaration of Helsinki (as revised in 2013). The study was approved by the Ethics Committee of The First Affiliated Hospital of Xiamen University and informed consent was taken from all the patients.

Open Access Statement: This is an Open Access article distributed in accordance with the Creative Commons Attribution-NonCommercial-NoDerivs 4.0 International License (CC BY-NC-ND 4.0), which permits the noncommercial replication and distribution of the article with the strict proviso that no changes or edits are made and the original work is properly cited (including links to both the formal publication through the relevant DOI and the license). See: https://creativecommons.org/licenses/by-nc-nd/4.0/.

\section{References}

1. Chen YP, Chan ATC, Le QT, et al. Nasopharyngeal carcinoma. Lancet 2019;394:64-80.

2. Sung H, Ferlay J, Siegel RL, et al. Global Cancer Statistics 2020: GLOBOCAN Estimates of Incidence and Mortality Worldwide for 36 Cancers in 185 Countries. CA Cancer J Clin 2021;71:209-49.

3. Ahmed Z, Kujtan L, Kennedy K, et al. The role of chemotherapy in the treatment of stage II nasopharyngeal carcinoma: Retrospective analysis of the national cancer database. Cancer Med 2019;8:1500-7.

4. Liao W, Tian M, Chen N. Characteristic And Novel Therapeutic Strategies Of Nasopharyngeal Carcinoma With Synchronous Metastasis. Cancer Manag Res 2019;11:8431-42.

5. Tseng PY, Liu YT, Lin CC, et al. Pinostilbene Hydrate Inhibits the Migration and Invasion of Human Nasopharyngeal Carcinoma Cells by Downregulating MMP-2 Expression and Suppressing EpithelialMesenchymal Transition Through the Mitogen-Activated Protein Kinase Signaling Pathways. Front Oncol 2019;9:1364.

6. Chai SJ, Ahmad Zabidi MM, Gan SP, et al. An Oncogenic Role for Four-Jointed Box 1 (FJX1) in Nasopharyngeal Carcinoma. Dis Markers 2019;2019:3857853.

7. Kim K, Eom J, Jung I. Characterization of Structural Variations in the Context of 3D Chromatin Structure. Mol Cells 2019;42:512-22.

8. Ing-Simmons E, Vaid R, Bing XY, et al. Independence of chromatin conformation and gene regulation during Drosophila dorsoventral patterning. Nat Genet 2021;53:487-99.

9. Van Berkum NL, Lieberman-Aiden E, Williams L, et al. Hi-C: a method to study the three-dimensional architecture of genomes. J Vis Exp 2010;(39):1869.

10. Goel VY, Hansen AS. The macro and micro of chromosome conformation capture. Wiley Interdiscip Rev Dev Biol 2020;e395.

11. Lee DI, Roy S. Graph-regularized matrix factorization for reliable detection of topological units from highthroughput chromosome conformation capture datasets. BioRxiv 2020. doi: 10.1101/2020.08.17.254615.

12. Fortin JP, Hansen KD. Reconstructing A/B compartments as revealed by Hi-C using long-range correlations in epigenetic data. Genome Biol 2015;16:180.

13. Kunz T, Rieber L, Mahony S. Assessing relationships between chromatin interactions and regulatory genomic activities using the self-organizing map. Methods 2021;189:12-21.

14. Lajoie BR, Dekker J, Kaplan N. The Hitchhiker's guide to $\mathrm{Hi}-\mathrm{C}$ analysis: practical guidelines. Methods 2015;72:65-75.

15. Wei Z, Gao F, Kim S, et al. Klf4 organizes longrange chromosomal interactions with the oct 4 locus in reprogramming and pluripotency. Cell Stem Cell 2013;13:36-47.

16. Servant N, Varoquaux N, Lajoie BR, et al. HiC-Pro: an 
optimized and flexible pipeline for $\mathrm{Hi}-\mathrm{C}$ data processing.

Genome Biol 2015;16:259.

17. Hu Z, Ma A, Tian H, et al. Effects of age on expression of BKca channel in vascular smooth muscle cells from mesenteric arteries of spontaneously hypertensive rats. J Physiol Biochem 2013;69:945-55.

18. Lieberman-Aiden E, van Berkum NL, Williams L, et al. Comprehensive mapping of long-range interactions reveals folding principles of the human genome. Science 2009;326:289-93.

19. Tanay A, Cavalli G. Chromosomal domains: epigenetic contexts and functional implications of genomic compartmentalization. Curr Opin Genet Dev 2013;23:197-203.

20. Dixon JR, Selvaraj S, Yue F, et al. Topological domains in mammalian genomes identified by analysis of chromatin interactions. Nature 2012;485:376-80.

21. Nora EP, Lajoie BR, Schulz EG, et al. Spatial partitioning of the regulatory landscape of the $\mathrm{X}$-inactivation centre. Nature 2012;485:381-5.

22. Mishiro T, Ishihara K, Hino S, et al. Architectural roles of multiple chromatin insulators at the human apolipoprotein gene cluster. EMBO J 2009;28:1234-45.

23. Dekker J, Mirny L. The 3D Genome as Moderator of Chromosomal Communication. Cell 2016;164:1110-21.

24. Dowen JM, Fan ZP, Hnisz D, et al. Control of cell identity genes occurs in insulated neighborhoods in mammalian chromosomes. Cell 2014;159:374-87.

25. Repana D, Nulsen J, Dressler L, et al. The Network of Cancer Genes (NCG): a comprehensive catalogue of known and candidate cancer genes from cancer sequencing screens. Genome Biol 2019;20:1.

26. Huynh L, Hormozdiari F. TAD fusion score: discovery and ranking the contribution of deletions to genome structure. Genome Biol 2019;20:60.

27. Wang J, Xie GF, He Y, et al. Interfering Expression of Chimeric Transcript SEPT7P2-PSPH Promotes Cell Proliferation in Patients with Nasopharyngeal Carcinoma. J Oncol 2019;2019:1654724.

28. Zhang Y, McCord RP, Ho YJ, et al. Spatial organization of the mouse genome and its role in recurrent chromosomal translocations. Cell 2012;148:908-21.

29. Zhang Z, Zhang X, Radiotherapy DO. The expression of HOXC6 in nasopharyngeal carcinoma and its clinical signific ance. Journal of Modern Oncology 2020;28:43-8.

30. Lu Y, Liang Y, Zheng X, et al. EVI1 promotes epithelialto-mesenchymal transition, cancer stem cell features and chemo-/radioresistance in nasopharyngeal carcinoma. J Exp Clin Cancer Res 2019;38:82.

31. Wang JY, Jin X, Li XF. Knockdown of TMPRSS3, a Transmembrane Serine Protease, Inhibits Proliferation, Migration, and Invasion in Human Nasopharyngeal Carcinoma Cells. Oncol Res 2018;26:95-101.

32. Sun K, Ye L, Chen Z, Zhou X. Correlation Analysis Between High Expression of HOXC10 and Prognosis of Nasopharyngeal Carcinoma Patients. Medical Journal of Wuhan University 2018;39:427-31.

33. Zhang J, Zheng ZQ, Yuan YW, et al. NFAT1 Hypermethylation Promotes Epithelial-Mesenchymal Transition and Metastasis in Nasopharyngeal Carcinoma by Activating ITGA6 Transcription. Neoplasia 2019;21:311-21.

34. Li J, Zhou C, Wang G, et al. Promoter hypermethylation of SLIT2 is a risk factor and potential diagnostic biomarker for nasopharyngeal carcinoma. Gene 2018;644:74-9.
Cite this article as: Yang Y, Chen M, Cheng L, Su C, Liao X, He H, You M, Rui G, Hong G. High-throughput chromosome conformation capture-based analysis of higher-order chromatin structure in nasopharyngeal carcinoma. Ann Transl Med 2021;9(16):1314. doi: 10.21037/atm-21-3273 